\title{
QUASI TWO-DIMENSIONAL NUCLEON SUPERFLUIDITY UNDER LOCALIZATION WITH PION CONDENSATION
}

\author{
TATSUYUKI TAKATSUKA \\ Faculty of Humanities and Social Sciences, Iwate University \\ Morioka 020-8550, Japan \\ takatuka@iwate-u.ac.jp
}

\begin{abstract}
The aim here is to show an example for localization and relevant low-dimensional superfluids in nuclear system. Due to a particular property of tensor force originating from the One-Pion-Exchange (OPE) between two nucleons, dense nuclear medium undergoes a layer confinement of the nucleons on one hand and also pion condensation (PC) for pion field mediating two-nucleon interaction on the other hand. The localization is characterized by a layered structure with a specific spin-isospin ordering. In that situation, the pairing problem has a two-dimensional (2D) character, i.e., low-dimensional superfluid realized in the hadronic matter with strong interactions. The pairing description suitable to the 2D nature is presented and possible realization of superfluidity in neutron stars is discussed, togeter with its effect on the cooling scenarios.
\end{abstract}

Keywords: Localization; Superfluidity; Neutron stars.

\section{Tensor Force and Localization}

Historically, study on localization in nucleon matter was initiated by a motivation to explain giant glitches of Vela pulsar through a starquake ${ }^{1}$ (corequake $^{2}$ ) made possible if the NS has a solid core. Realistic calculations@ ${ }^{3,4}$, however, have shown that the short-range repulsion of two-nucleon interaction $V(1,2)$ is not so steep as to realize the quantum solid through the geometrical caging mechanism, as compared to the He system. In this context, it is of particular interest to ask whether another solidification mechanism is existent or not in nuclear medium. The answer is 'yes ${ }^{{ }^{5-7}}$. We pay attention to the noncentral character of $V(1,2)$ which consists of the central, spin-orbit and tensor components;

$$
\begin{array}{r}
V(1,2)=V_{C}(r)+V_{L S}(r) \mathbf{L} \cdot \mathbf{S}+V_{T}(r) S_{12}, \\
S_{12}=3\left(\boldsymbol{\sigma}_{1} \cdot \hat{\mathbf{r}}\right)\left(\boldsymbol{\sigma}_{2} \cdot \hat{\mathbf{r}}\right)-\left(\boldsymbol{\sigma}_{1} \cdot \boldsymbol{\sigma}_{2}\right),
\end{array}
$$

with $S_{12}$ the tensor operator depending on the spin $(\boldsymbol{\sigma})$ and direction $(\hat{\mathbf{r}})$. For a parallel-spin pair, we have the diagonal spin matrix element $\left\langle S_{12}\right\rangle=\left(3 \cos ^{2} \theta-1\right)$ and see that for the isotriplet state of a $n n$ or $p p$ pair (the isosinglet state of a $n p$ pair), the $\theta \simeq \pi / 2(\theta \simeq 0)$ direction is energetically most favorable relative position 


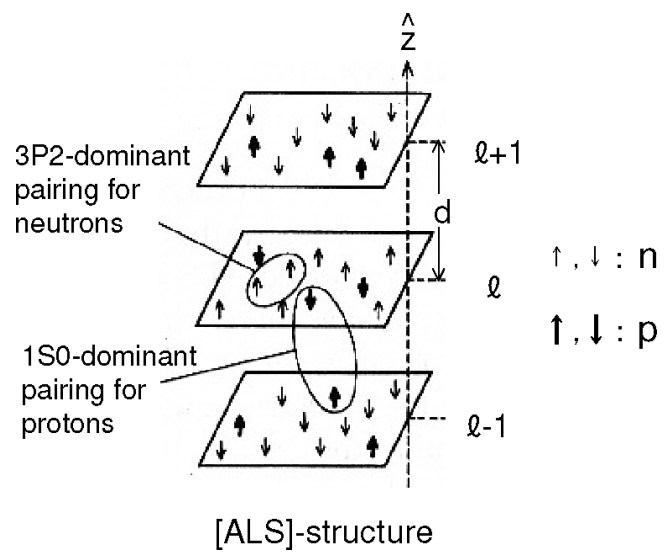

Fig. 1. ALS structure with one-dimensional localization and specific spin-isospin ordering.

because of $V_{T}>0\left(V_{T}<0\right)$. Here $\theta$ is the angle between the spin quantization axis ( $z$-direction) and direction $\hat{\mathbf{r}}$ with $\mathbf{r}=\mathbf{r}_{1}-\mathbf{r}_{2}$. Similarly for an antiparallel-spin pair, $<S_{12}>=\left(-3 \cos ^{2} \theta+1\right)$ means that the $\theta \simeq 0(\theta \simeq \pi / 2)$ is most favorable for a $n n$ or $p p$ pair ( $n p$ pair).

We remark the points; (i) In usual Fermi gas (FG) phase, the first-order effects from tensor force vanish as a whole. (ii) In order to utilize the tensor force effects attractively, the specific spin-isospin ordering and at the same time, the localization of nucleons are necessary. (iii) Thus we have a new solidification (localization) mechanism if the nuclear system with (ii) is energetically favorable compared to FG phase.

\section{ALS Model and Pion Condensation}

From a viewpoint of large kinetic energy (KE) increase due to localization ( $3 \hbar \omega / 4>\mathrm{KE}$ of $3 \mathrm{D}-\mathrm{FG})$, the one-dimensional (1D) localization, instead of usual $3 \mathrm{D}$ one, is advantageous because of smaller zero-point energy and is found not to lose the energy gain from interaction. Thus we propose an interesting structure of nucleon matter illustrated in Fig.1, called Alternating-Layer-Spin (ALS) structure taking account of the tensor force attraction most efficiently ${ }^{6}$. It is characterized by a layered structure due to 1D-localization and by a specific spin-isospin ordering, i.e., in the $\ell$-th layer, the spin of like nucleons are aligned in the combination $(\mathrm{n} \uparrow$, $\mathrm{p} \downarrow)$ and the spin directions change alternately layer by layer.

In the ALS model, the single-particle state is given by the following orthogonal basis functions $\left\{\phi_{\alpha}(\xi)\right\}$;

$$
\phi_{\alpha}(\boldsymbol{\xi}) \equiv \phi\left(\boldsymbol{\xi} ; \mathbf{q}_{\perp}, \ell\right)=\exp \left(i \mathbf{q}_{\perp} \mathbf{r}_{\perp}\right) / \sqrt{\Omega_{\perp}} \cdot \phi_{\ell}(z) \chi_{\alpha}(\text { spin, isospin }),
$$

where $\boldsymbol{\xi} \equiv(\mathbf{r}$, spin, isospin $), \mathbf{q}_{\perp} \equiv\left(q_{x}, q_{y}\right), \mathbf{r}_{\perp} \equiv(x, y), \Omega_{\perp}$ is the 2D-normalization volume and the suffix $\alpha$ of the spin-isospin function $\chi$ means the combination of 
$\left(\sigma_{\ell}, \tau_{\ell}\right)$ as $\sigma_{\ell} \tau_{\ell}=-(-1)^{\ell}, \sigma_{\ell}(n)=(-1)^{\ell}$ and $\sigma_{\ell}(p)=(-1)^{\ell+1}$ with $\tau_{\ell}=+1(-1)$ for $\mathrm{p}(\mathrm{n}) . \phi_{\ell}(\mathrm{z})$ is given by the Bloch or Wannier function and is well approximated by a Gaussian function, $\phi_{\ell}(z)=(a / \pi)^{1 / 4} \exp \left[-a(z-d \ell)^{2} / 2\right]$ for a developed localization, with $d$ the layer spacing.

How about the pion field $\phi$ in the ALS structure? Due to the 1D localization and spin-isospin ordering, we have a periodic spin-isospin density wave which provides a static source function $S(\mathbf{r})$ in $z$-direction and thereby $\phi$ has a non-zero expectation value $(\langle\phi\rangle \neq 0)$ through the field equation for the $\pi-N P$-wave interaction $\left(H_{\pi N}\right.$ with $\sigma \cdot \nabla$-coupling),

$$
\left(\nabla^{2}-m_{\pi}^{2}\right)<\phi>=-\left(f / m_{\pi}\right) \nabla \mathbf{S}(\mathbf{r})
$$

The non-vanishing $\langle\phi\rangle \neq 0$ means that pions participate as a real component of the medium owing to a particular structure of nucleon system, in contrast to $\langle\phi\rangle=0$ for the usual FG state. This situation is nothing but the pion condensation (PC) which has gathered much attention since the first proposal by A.B. Migdal ${ }^{8}$ and independently by R.F. Sawyer and D.J. Scalapino ${ }^{9}$. The condensed pion field (in the present case, neutral pion field $\left\langle\phi_{0}>\right.$ as seen from Eq. (4)) has a form of a standing wave mode $<\phi_{0}>\propto \sin k_{0} z$ with the condensed momentum $k_{0}=$ $\pi / d$. The equivalence between ALS structure and neutral pion $\left(\pi^{0}\right)$ condensation has been shown through the equivalence between field description and potential description $^{6,7,10}$.

The ALS structure (i.e., $\pi^{0}$ condensation) is realized when the condensation energy (attractive contribution from OPE tensor) overwhelms the kinetic energy increase due to localization $(=\hbar \omega / 4+\mathrm{KE}$ of $2 \mathrm{D}-\mathrm{FG}-\mathrm{KE}$ of $3 \mathrm{D}-\mathrm{FG})$. The onset density $\rho_{t}\left(\pi^{0}\right)$ is estimated as $\rho_{t}\left(\pi^{0}\right) \simeq(2-4) \rho_{0}$, with $\rho_{0}\left(=0.17\right.$ nucleons $/ \mathrm{fm}^{3} \simeq$ $2.8 \times 10^{14} \mathrm{~g} / \mathrm{cc}$ ) being the nuclear density.

So far we have concentrated on the $\pi^{0}$ condensation. When we pay our attention to the isospin degrees of freedom of the pion field, we have energetically more favorable structure by allowing the coexistence of $\pi^{0}$ - and $\pi^{c}$ (charged pion)condensations $^{7}$. We extend the ALS model in such a way as the $\pi^{c}$ condensation of a running wave mode is realized within the ALS layer $(x-y$ plane) with the condensed momentum $\mathbf{k}_{c}$ in $\mathbf{r}_{\perp}$ direction perpendicular to $\mathbf{k}_{0}$ in the $z$-direction. In this case, the nucleon basis function is given by a superposition of $n$ - and $p$-ALS wave functions and the ALS structure corresponding to the combined neutral and charged pion condensations $\left(\pi^{0} \pi^{c}\right.$ condensation) is given likely in Fig. 1 with a replacement of $n$ by $\eta$, called 'new neutron' $\left(|\eta>=u| n>+v \mid p>, u^{2}+v^{2}=1\right)$ including $n$ as a dominant component. A remarkable point is that both condensations are realized without serious interference and the energy gains from them are additive. Thus the ground state for nucleon matter with PC is given by the extended ALS model with $\pi^{0} \pi^{c}$ condensation. By a realistic treatment, the onset density $\rho_{t}\left(\pi^{0} \pi^{c}\right)$ has been estimated as $\rho_{t}\left(\pi^{0} \pi^{c}\right) \simeq(2.5-4) \rho_{0}{ }^{12}$. 


\section{Low-Dimensional Superfluidity}

According to the increase of $\rho$, the nuclear lattice in the crust phase of NSs tends to resolve and at $\rho \gtrsim 0.5 \rho_{0}$ the matter is in a uniform system, called 'liquid core', primarily composed of $n$ as a main component and $p$ by several $\%$ and also leptons $\left(e^{-}, \mu^{-}\right)$assuring the charge neutrality. In nucleon matter, the most attractive pair state responsible for a realization of superfluidity depends on the fractional density $\rho_{i}(i=n, p)$. Here a pair state is specified by a set of quntum numbers $\tilde{\lambda} \equiv(T, S, L, J)$ with $T, S, L$ and $J$ being the pair's isospin, spin, orbital and total angular momenta, respectively, due to characteristics of nuclear force $V(1,2)$ in Eq. (1). In the case of $p p$ pair near the Fermi surface, the scattering energy $E_{N N}^{L A B}\left(=4 \times\right.$ Fermi energy $\left.=4 \epsilon_{i F} \simeq 244\left(\rho_{i} / \rho_{0}\right)^{2 / 3}, i=n, p\right)$ is low because of small contamination $\left(\rho_{p}<<\rho\right)$ and the ${ }^{1} S_{0}$ pair state, most attractive at low $E_{N N}^{L A B}$, should be responsible for superfluidity. On the other hand, the ${ }^{3} P_{2}$ pair state, most attractive at high $E_{N N}^{L A B}$, should be responsible for neutron superfluidity, dominance in components $\left(\rho_{n} \simeq \rho\right)$ and high $E_{N N}^{L A B}$. That is, the ${ }^{1} S_{0}$-superfluidity for $p$ and the ${ }^{3} P_{2}$-one for $n$ are expected. The former is a well known type as a $S$-state superfluidity, but the latter is a new one. This possibility of the ${ }^{3} \mathrm{P}_{2}$-superfluid was firstly pointed out by R. Tamagaki ${ }^{13}$ and also by M. Hoffberg et al. ${ }^{14}$, which was prior to the discovery of P-state superfluid of ${ }^{3} \mathrm{H}_{e}$ system in 1972. Realistic investigations by $\mathrm{R}$. Tamagaki and myself ${ }^{15-17}$ led to a conclusion that the $\mathrm{n}^{3} \mathrm{P}_{2^{-}}$ superfluid is realized in the density region $\rho \simeq(1-4) \rho_{0}$ of NSs.

Now we go to the superfluid problem under ALS structure. Since the above existence-region of the ${ }^{3} \mathrm{P}_{2}$ superfluid overlaps that of the ALS phase, our main concern is whether the ${ }^{3} \mathrm{P}_{2}$-superfluidity persists or not in the ALS localization. Due to 1D localization, the Fermi surface turns out to be of cylindrical shape and the single-particle energy is expressed as $\epsilon\left(q_{\perp}\right)=\hbar^{2} q_{\perp}^{2} / 2 m_{N}^{*}+$ const. with $m_{N}^{*}$ the effective mass. The pairing correlation for the $\left(\mathbf{q}_{\perp} \ell,-\mathbf{q}_{\perp} \ell \mathbf{f}\right)$-Cooper pair is operative in the $\mathbf{r}_{\perp}$-space where the $2 \mathrm{D}$ Fermi gas nature holds. To see what pair state is most attractive, the $2 \mathrm{D}$ plane wave of the pair wave function is expanded as ${ }^{18}$

$$
e^{i \mathbf{q}_{\perp} \mathbf{r}_{\perp}}=\sum_{m_{L}}(i)^{m_{L}} J_{m_{L}}\left(q_{\perp} r_{\perp}\right) e^{i m_{L} \varphi_{r}} e^{-i m_{\perp} \varphi_{q}}
$$

where partial waves are specified by $m_{L}$ (the $2 \mathrm{D}$ angular momentum). Localization makes the pairing interaction in the same layer predominant and for simplicity an approximation to ignore the contribution from the pairs with $\ell \neq \ell^{\prime}$ is taken. Then the spin part of the wave function is characterized by $S=1$ and $m_{S}=(-)^{\ell}(z-$ component of $S$ ) due to the spin alignment. Finally, a pair state in the ALS scheme is specified by a set of quantum numbers $\tilde{\lambda}=\left\{S=1, m_{S}=(-)^{\ell}, m_{L}\right\}$ instead of $\lambda$ of $3 \mathrm{D}$ case.

What $\tilde{\lambda}$-pair is most attractive? We construct the $2 \mathrm{D}$ effective potential $\tilde{V}_{\tilde{\lambda}}\left(r_{\perp}\right)$ by taking average of $V(1,2)$ over the localized wave $\phi_{\text {rel }}(\mathrm{z}) \equiv(a / 2 \pi)^{1 / 4} \exp \left(-\mathrm{az}^{2} / 4\right)$ 
as

$$
V_{\tilde{\lambda}}\left(r_{\perp}\right) \equiv<\tilde{\lambda}|V(1,2)| \tilde{\lambda}>=\tilde{V}_{c}\left(r_{\perp}\right)+m_{S} m_{L} \tilde{V}_{L S}\left(r_{\perp}\right)+\tilde{V}_{T}\left(r_{\perp}\right) .
$$

Eq.(6) indicates that $V_{\tilde{\lambda}}\left(r_{\perp}\right)$ is more attractive for the pair with $m_{L} m_{S}=m_{L}(-)^{\ell}>$ 0 because of $\tilde{V}_{L S}\left(r_{\perp}\right)<0$. Consequently we should take the combination; $m_{L}=1$, $3, \ldots$ for $m_{S}=1$ ( $\ell=$ even layers $)$ and $m_{L}=-1,-3, \ldots$ for $m_{S}=-1(\ell=$ odd layers $)$, with the restriction $\left|m_{L}\right|=1$ due to antisymmetrization. Among possible $m_{L},\left|m_{L}\right|=1$ is most effective due to the centrifugal effects. The state with $\tilde{\lambda} \equiv\left\{S=1, m_{S}=\right.$ $m_{L}=1$ or $\left.m_{S}=m_{L}=-1\right\}$ contains the contribution from the ${ }^{3} P_{2}$-interaction as a main contributor and is the most attractive pair state in the $\tilde{\lambda}$ scheme at high densities. This pairing is suitably called ' ${ }^{3} P_{2}$-dominant pairing' ${ }^{18}$. The energy gap equation for the ${ }^{3} P_{2}$-dominant pairing has a $2 \mathrm{D}$ character in the points; $2 \mathrm{D}$ interaction in Eq. (6), 2D single-particle energy $\epsilon\left(q_{\perp}\right)$ and the 2D momentum space. Numerical calculations of the energy gap show that the ${ }^{3} P_{2}$-superfluidity in usual $3 \mathrm{D}$ case can persist as a ${ }^{3} P_{2}$-dominant superfluidity of $2 \mathrm{D}$ character, even if the $1 \mathrm{D}$ ALS-localization comes into play in NS cores. By the way, the proton ${ }^{1} S_{0}$-dominant superfluidity (superconductivity) can be treated quite similarly.

However this conclusion is for a simple case where pure $n n$-pair and ALS-phase only with $\pi^{0}$ condensation are taken into account. More realistic discussions need to include the following points; (i)As has been discussed in the preceding section, the ground state with PC should be described by the extended ALS with a coexistence of $\pi^{0}$ and $\pi^{c}$ condensations. (ii) Isobar $\Delta$ effects important for the realization of ALS. The point (i) requires that the pairing correlation has to be treated on the basis of $\eta$-particles. In this case, the attraction for the ${ }^{3} P_{2}$-dominant pairing is weakened due to the fact that the $\eta$-state is a superposition of $n$ and $p$ states, which is called 'attenuation effect'and works against the occurrence of superfluidity. For the point (ii), the $\eta$-particle state is extended as a quasi-baryon state given by a superposition of nucleon and isobar states and the BCS state due to the ${ }^{3} \mathrm{P}_{2^{-}}$ dominant pairing is treated on this quasi-baryon baisis. In this case, the attraction is enhanced mainly through the enhancement of $\tilde{V}_{T}\left(r_{\perp}\right)$ in Eq.(6), working for the realization of superfluidity. As a net effects from (i) and (ii), the ${ }^{3} \mathrm{P}_{2}$-dominant superfluidity of quasibaryons is found to occur in NS $\operatorname{cores}^{19}$, with the critical temperature $T_{c} \sim(0.5-2) \times 10^{9} \mathrm{~K}$ exceeding the internal temperature $T_{i n} \simeq 10^{8} \mathrm{~K}$ of NSs.

\section{Concluding Remarks}

We want to stress that a layered structure of nucleon matter, a solid-like state of $1 \mathrm{D}$ localization with a specific spin-isospin ordering, is possible due to the characteristics of nuclear force (tensor force), which is nothing but the pion-condensed state in the field description. The system is well described by the ALS model. It is remarked that under the situation the pairing correlation becomes of a $2 \mathrm{D}$ character, presenting an interesting example of low-dimensional superfluid in hadronic matter with strong interactions. 
Finally we want to stress the importance of superfluidity in a context of NS cooling. Observed colder class NSs( such as Vela, Geminga, 3C58 and so on) cannot be explained by a standard cooling process (modified URCA) but encounter the problem of 'too rapid cooling'when rapid cooling process (extremely efficient $\nu$ emission by usual $\beta$-decay type in exotic phases) is applied, that is, require the rapid cooling and the superfluidity to suppress too rapid cooling, at the same time. In this sense, the so-called 'Pion cooling'( $\beta$-decay of $\eta$-particles) is a promising candidate, since both requirements above are satisfied by the ALS-phase with PC. It is wortwhile to note that this pion cooling necessarily shows up as a unique candidate, because another promising one, the so-called 'hyperon cooling'(hyperon $\beta$-decay in hyperon-mixed NS cores), though successfully applied, is faced to a serious difficulty as to the occurence of $\Lambda$-superfluidity ${ }^{20}$.

\section{Acknowledgments}

The author expresses a special thank to R. Tamagaki for continueous collaboration on the series of work presented here. He also thanks S. Tsuruta, T. Tatsumi, T. Muto, S. Nishizaki and J. Hiura for useful discussions and encouragements.

\section{References}

1. G. Baym, C.J. Pethick, D. Pines and M. Ruderman, Nature 224, 872 (1969).

2. D. Pines, J. Shaham and M. Ruderman, Nature Phys. Sci. 237, 83 (1972).

3. D.M. Ceperley, G.V. Chester and M.H. Kalos, Phys. Rev. D13, 3208 (1976).

4. D.N. Lowy and C-. W. Woo, Phys. Rev. D13, 3201 (1976).

5. T. Takatsuka, K. Tamiya and R. Tamagaki, Prog. Theor. Phys. 56, 685 (1976).

6. T. Takatsuka, K Tamiya, T. Tatsumi and R. Tamagaki, Prog. Theor. Phys. 59, 1933 (1978).

7. T. Takatsuka, R. Tamagaki and T. Tatsumi, Prog. Theor. Phys. Suppl. No. 112,67 (1993).

8. A.B. Migdal, Zh. Exsper. Theor. Fiz. 63, 1993 (1972); Sov. Phys. JETP 36, 1052 (1973).

9. R.F. Sawyer and D.J. Scalapino, Phys. Rev. D7, 953 (1972).

10. T. Takatsuka and J. Hiura, Prog. Theor. Phys. 60, 1234 (1978).

11. T. Kunihiro, T. Takatsuka, R. Tamagaki and T. Tatsumi, Prog. Theor. Phys. Suppl. No. 112, 123 (1993).

12. T. Muto, R. Tamagaki and T. Tatsumi, Prog. Theor. Phys. Suppl. No. 112, 159 (1993).

13. R. Tamagaki, Prog. Theor. Phys. 44, 905 (1970).

14. M. Hoffberg, A.E. Glassgold, R.W. Richardson and M. Ruderman, Phys. Rev. Lett. 24, 775 (1970).

15. T. Takatsuka and R. Tamagaki, Prog. Theor. Phys. 46, 114 (1971).

16. T. Takatsuka, Prog. Theor. Phys. 48, 1517 (1972).

17. T. Takatsuka and R. Tamagaki, Prog. Theor. Phys. 112, 37 (2004).

18. T. Takatsuka and R. Tamagaki, Prog. Theor. Phys. Suppl. No. 112, 107 (1993).

19. R. Tamagaki and T. Takatsuka, Prog. Theor. Phys. 116, 573 (2006); 117, 861 (2007).

20. S. Tsuruta, J. Sadino, A. Kobeiski, M.A. Teter, A.C. Liebmann, T. Takatsuka, K. Nomoto and H. Umeda, Ap. J. 691, 691 (2009). 